\title{
La Gamificación como Herramienta Tecnológica para el Aprendizaje en la Educación Superior
}

\author{
Gamification as a Technological Tool for Learning in Higher Education
}

Berenice Del Carmen Heredia-Sánchez $z^{1}$, Damaris Pérez-Cruz², Jose Felipe Cocón-Juárez ${ }^{3}$ y Patricia Zavaleta-Carrillo

\section{()}

\section{EDICIÓN: @e-CIVTAC}

Recibido: 6/julio/2020

Aceptado: 6/agosto/2020

Publicado: 25/septiembre/2020

\section{我 ${ }^{1}$ México \\ México \\ ${ }^{3}$ México \\ ${ }^{4}$ México}

\section{IIIIInstitución}

${ }^{1}$ Universidad Autónoma Del Carmen Universidad Autónoma Del Carmen ${ }^{3}$ Universidad Autónoma Del Carmen ${ }^{4}$ Universidad Autónoma Del Carmen

\section{Correo Eletrónico}

1bcheredia@hotmail.com 2dperez@pampano.unacar.mx 3jcocon@pampano.unacar.mx ${ }^{4}$ pzavaleta@pampano.unacar.mx

\section{(1) ORCID}

${ }^{1}$ https://orcid.org/0000-0002-1392-4750 ${ }^{2} \mathrm{https} / / /$ orcid.org/0000-0002-6226-9561 ${ }^{3}$ https://orcid.org/0000-0002-6932-683X ${ }^{4} \mathrm{https}$ ://orcid.org/0000-0003-2001-6950

\section{Citar así: GAPA / IEEE}

Heredia-Sánchez, B., Pérez-Cruz, D., Cocón-Juárez , J. \& Zavaleta-Carrillo, P (2020). La Gamificación como Herramienta Tecnológica para el Aprendizaje en la Educación Superior. Revista Tecnológica-Educativa Docentes 2.0, 9(2), 49-58. https://doi.org/10.37843/rted.v9i2.144

B. Heredia-Sánchez, D. Pérez-Cruz, J. Cocón-Juárez y P. Zavaleta-Carrillo, "La Gamificación como Herramienta Tecnológica para el Aprendizaje en la Educación Superior", RTED, vol. 9, n. ${ }^{\circ} 2$, pp. 49-58, sep. 2020.

\section{Resumen}

Esta investigación presentó la gamificación como una tecnología innovadora que el docente puede aplicar en las asignaturas del nivel educativo superior, con el propósito de aumentar el interés de los estudiantes por el estudio, y que se vea reflejado en el rendimiento académico de los universitarios. El avance de la tecnología del juego y entrenamiento ha alcanzado a la educación de tal manera que actualmente el profesor tiene que buscar estrategias y herramientas para que el proceso de aprendizaje sea más interesante para los estudiantes. Es por ello, que con este objetivo se realizó la investigación de diferentes herramientas actuales de gamificación con impacto en el aula como una estrategia eficaz para el aprendizaje de las asignaturas. El enfoque cualitativo nos permite aplicar una investigación documental-bibliográfica, facilitando la revisión y comparación de tres herramientas, para determinar cuál ofrece mayores beneficios, tanto a docentes, como a los estudiantes, por tanto, se concluye que la herramienta llamada Quizizz es la más completa. El uso de esta herramienta, de la mano de la creatividad del docente en el diseño de actividades significativas a manera de juego, mejora el desempeño y los resultados de los estudiantes en las asignaturas. Sin embargo, hay que tomar en cuenta que la medida de eficiencia de los resultados obtenidos no solo depende de las herramientas, considerando otros factores que pueden afectar estos resultados.

Palabras clave: Gamificación, herramientas de gamificación, tecnologías de la información.

\begin{abstract}
This research presents gamification as an innovative technology that the teacher can apply in the subjects of the higher educational level, with the purpose of increasing the interest of the students in the study, and what to see reflected in the academic performance of the university students. The advancement of game and training technology has brought education in such a way that currently the teacher has to find strategies and tools for the most interesting sea learning process for students. That is why, with this objective, the investigation of different current gamification tools with impact in the classroom was carried out as an effective strategy for learning the subjects. The qualitative approach allows us to apply a documentary-bibliographic research, facilitate the review and comparison of three tools, determine what offers the greatest benefits, both to teachers and students, therefore, it is concluded that the tool called Quizizz is the most Complete The use of this tool, hand in hand with the creativity of the teacher in the design of dynamic activities as a game, improves the performance and results of the students in the subjects. However, it must be considered that the efficiency measure of the affected results does not only depend on the tools, other factors that may affect these results.
\end{abstract}

Keywords: Gamification, gamification tools, information technologies. 


\section{Introducción}

¿A qué problemas se enfrenta el docente en enseñanza de diferentes asignaturas en la educación actual? Hoy en día vivimos en mundo en continuo estado de transformación, en el que la tecnología moderna puede ayudar al docente a ofrecer diversas estrategias mediante las nuevas tecnologías particularmente útiles que motivan a la elaboración de actividades en aulas de educación superior (Carrión Candel, 2018, pág. 2). Siendo la tecnología un instrumento de condición humana que permita diseñar todo aquello con el fin de perfeccionar su trabajo y la mejora de la calidad de su entorno social (Lopez-Jiménez, 2007, p.73-74).

Una de las dificultades que se presenta hoy en día en la enseñanza, particularmente en las aulas de nivel superior, es el aprendizaje de los alumnos, a causa de que los docentes recurren al material convencional basado en libros de texto lo que propicia que el alumno percibe la información desde su asiento. En el ámbito universitario Olivia (2017) considera de gran utilidad la estrategia de gamificación aplicada en el contexto universitario, lo que permite que la clase pueda superar la conjugación de conocimiento y la estructura del juego pueda generar el interés y compromiso del estudiante.

Por otra parte, se puede resaltar que el uso de las tecnologías en los últimos años se ve implicada en el rendimiento de los alumnos, por lo que, se debe aprovechar el uso de esos dispositivos en las aulas para que el alumno mejore su aprendizaje y no decaiga su interés con el pasar del tiempo. Por lo tanto, la tecnología educativa viene a reforzar el proceso de enseñanza-aprendizaje fortaleciendo el aprendizaje y mejorando la calidad en la enseñanza con el empleo de medios de audio, vídeo e imagen que son de gran dominio en los jóvenes estudiantes (Mujica, 2020, p.3).

Considerando lo que menciona Müller (2019) nos encontramos en una educación en dónde el rol del docente se convierte un orientador cuya primordial tarea es guiar el aprendizaje de sus alumnos promoviendo el aprendizaje autónomo a través de aprendizaje basado en proyectos, preparación de micro actividades motivantes fuera de clase (flipped classroom), estudio de casos, entre otros, favoreciendo al aprendizaje autodidacta, como método de aprendizaje.

De esta manera el alumno lleva un conocimiento previo que se traducirá en un mejor aprovechamiento de su tiempo en horas de clases y el profesor puede realizar actividades para potencializar el aprendizaje del alumno. Este es el reto de la educación superior, que los profesores conozcan las capacidades y deficiencias de los jóvenes para orientarlas en desarrollo de habilidades a través de actividades utilizando los recursos tecnológicos digitales, de comunicación y virtualidad para cumplir las expectativas que demanda la formación del nuevo profesionista (Rodríguez, 2010, p.1).

\section{Inicios de la Gamificación}

Los inicios de la gamificación se dieron a través del sector empresarial en el año 1896, donde una empresa vendía estampas a otras empresas minoristas utilizadas para recompensar a sus clientes y así poder beneficiar a la empresa e incentivar al cliente. Sin embargo, Nick Pelling acuña al término por primera vez en el año 2003 "La gamificación como la aplicación de metáforas de juego para tareas de la vida real que influyen en el comportamiento y mejora la motivación y el compromiso de las personas que se implican en estos procesos gamificados", en donde se fueron adaptando a diferentes ámbitos y los fueron difundiendo en congresos y conferencias (Rodríguez \& Santiago Campión, 2015, pág. 8).

Por tanto, la educación 3.0 considera que la gamificación es una técnica o estrategia de aprendizaje que traslada el funcionamiento del juego a un entorno educativo o profesional con el objetivo de alcanzar mejores resultados Palazón (2019), coincide con lo que menciona Deterding (2011) y lo que propone Ara-Moreira y González-González (2015) sobre aprovechar la adicción de los jóvenes por los videojuegos en lo referente a la necesidad de llevar el reconocimiento y los logros de lo real, a nuevos entornos digitales haciendo una asociación entre elementos de gamificación vs beneficios en el aprendizaje.

Por otra parte, los inicios de la gamificación en el ámbito educativo se originaron por Sawyer y Smith quienes aplicaron la taxonomía de no solo hacer juegos para diversión sino también para aplicarlos a diferentes sectores ya sea desde una formación, educación y salud (Vergara-Rodriguez \& Gómez-Vallecillo, 2017, p.1). 
Sin embargo, fue hasta el 2013 cuando la tecnología empezó aplicarse a la gamificación considerando que tiene aportes sustanciales en el aprendizaje (Green \& Bavelier, 2006, p.3-4).

De acuerdo con Gálvez y Rodríguez, el juego es primordial en las primeras etapas de vida y con la incorporación de nuevas tecnologías se logra un gran impacto en un marco educativo. (Gálvez Sosa \& Rodríguez Ledezma, 2005, pág. 33). Los primeros juegos con tecnologías que incorporaron los docentes para enseñar a sus alumnos fueron Trivinet, ClassDojo, Goalbook, Classcraft, Testeando, entre otros.

Hoy en día existen variedad de juegos y aplicaciones con Tecnologías de Información y la Comunicación (TIC) que pueden ser aprovechadas para enseñar con el propósito de motivar a los alumnos en su propio aprendizaje. Por otro lado, también son indispensables para motivar a los docentes en el desarrollo de actividades divertidas, amenas, creativas y estratégicas que contribuyan a elevar el interés por el estudio y conlleven a mejores resultados en el rendimiento académico de los alumnos. A continuación, se enlistan las herramientas más usadas actualmente (Palazón, 2020, p.1):
1. Knowre
2. Cerebriti
3. Kahoot
4. Socrative
5. Quizizz.
6. Brainscape

\section{Metodología}

El desarrollo de este artículo se desprende de un enfoque de investigación cualitativa, por sus características de planteamientos más abiertos, no se fundamenta en la estadística, además, permite la profundidad de significados, riqueza interpretativa, contextualización del fenómeno entre otras bondades (Hernandez-Sampieri, Fernández-Collado, \& Baptista-Lucio, 2014); con el propósito de investigar cuáles son las herramientas tecnológicas de gamificación que facilitan la enseñanza en aulas a nivel universitario y con lleven a mejorar el rendimiento académico. Los pasos para investigar estas nuevas tecnologías son los siguientes:

4. Hacer un análisis de las herramientas que ayudarían a mejorar la gamificación en la educación superior.
1. educación superior.

2. Identificar qué herramientas son opciones relevantes para la comparación de elementos.

3. Realizar el análisis de las herramientas seleccionadas y seleccionar una como resultado final.

Siguiendo estos pasos se realizó una búsqueda de materiales documentales (artículos de investigación, artículos de páginas web, blogs, entre otros) en donde se hace referencia a herramientas para gamificación. Posteriormente, desde el punto de vista del rol de docente, se determinaron ciertos elementos de comparación para encontrar aquellas con un alto potencial de uso en las aulas y, al mismo tiempo facilite el proceso de enseñanza-aprendizaje.

Con este trabajo se busca ofrecer a los docentes, otras herramientas tecnológicas que les permitan realizar sus clases más entretenidas, aumentar el interés del alumno por su aprendizaje y al mismo tiempo incrementar la motivación para asistir a clases.

\section{Análisis de Herramientas}

Dentro de la metodología en esta investigación se realizaron las siguientes actividades para poder llegar a un resultado final:

1. Se hizo un seleccionado de herramienta en donde se llevó a cabo una búsqueda profunda de herramientas web con disponibilidad para dispositivos móvil, tomando en cuenta de que hoy en día los celulares son los dispositivos más usados y que una gran cantidad de alumnos cuenta con uno.

2. Se fueron eligiendo cuáles herramientas contaban con ciertas características que fueran de utilidad para el docente para aprovechar al máximo su uso y principalmente que entrara en la categoría de la gamificación.

3. Dentro de la investigación se obtuvieron tres herramientas que son relevantes en el ámbito educativo por que cuentan con características importantes y se pueden llegar a comparar entre ellas mismas.

Lo anterior, nos llevó a considerar a Kahoot, Socrative y Quizziz, debido a que son herramientas con bondades en pro de una educación divertida, 
integral y motivadora en los estudiantes. En el caso de los profesores ofrecen invertir el tiempo-esfuerzo requeridos en la elaboración de actividades y se vea reflejado en creatividad didáctica enfocada al desarrollo de habilidades o necesidades individuales de los estudiantes. Para la evaluación de estas herramientas se realizaron dos actividades: 1) Analizar todas las funcionalidades en su versión gratuita y, 2) Explicar las diferentes características con los que cuenta la herramienta.

Finalmente, para poder realizar el análisis se determinó los atributos que serán elementos comparativos en las tres herramientas, estos atributos de muestran en la Tabla 1.

\section{Tabla 1}

Atributos evaluables en las herramientas de Gamificación.

\begin{tabular}{l}
\hline \multicolumn{1}{c}{ Listado de atributos } \\
\hline Acceso gratuito \\
\hline Restricciones en formato free \\
\hline Acceso a través de cuenta de Google \\
\hline Disponible para Android e iOS \\
\hline Acceso en el tiempo \\
\hline Cuenta o acceso como alumno \\
\hline Misma clave o pin de acceso para alumno \\
\hline Opciones de estilos de preguntas \\
\hline Respuestas múltiples \\
\hline Control del tiempo en las preguntas \\
\hline Generar preguntas aleatorias \\
\hline Revisión de respuesta \\
\hline Catálogo o biblioteca de preguntas \\
\hline Ritmo de respuesta \\
\hline Integración de elementos multimedios \\
\hline Acceder computadora o a través de dispositivo móvil \\
\hline Reporte de dominio de preguntas \\
\hline Reporte de resultados de los cuestionarios \\
\hline $\begin{array}{l}\text { Nota. Tabla que lista los atributos requeridos en el análisis final } \\
\text { de las herramientas de esta investigación, elaboración propia. }\end{array}$
\end{tabular}

En los siguientes párrafos, se describe a detalle los atributos o características con que cuentan Kahoot, Socrative y Quizziz:

Kahoot. Esta herramienta es considerada en la web 2.0 como una herramienta para desarrollar actividades interactivas y divertidas a través de juegos de preguntas y respuestas de forma muy intuitiva y dinámica (Kahoot, 2013). Como profesor puedes crear tus propios cuestionarios o encuestas online, puedes asignar valoración a cada pregunta, puedes integrar pregunta con imágenes y videos, por último, generar un pin para compartir el cuestionario a los estudiantes. En el caso de los estudiantes sin requerir una cuenta, con solo proporcionar el pin generado por el profesor, podrán acceder desde cualquier dispositivo conectado a un navegador web, podrá resolver los cuestionarios, así como también conocer las puntuaciones obtenidas (en caso de tener puntuación). Esta herramienta promueve la competencia tecnológica, facilita la motivación e incrementa el compromiso y la implicación del estudiante en su propio proceso de aprendizaje (Kahoot, 2013). Su sitio de trabajo se encuentra en https://kahoot.com/schools-u/. A continuación, se muestran a detalle sus características haciendo énfasis en los atributos señalados en la Tabla 1:

1. Opciones de preguntas. En cuanto a variedad de tipos de respuesta, Kahoot ofrece 7 opciones de respuestas, sin embargo, sólo cuatro de esas opciones están disponibles para las versiones de paga como se muestra en la Imagen 1. Esta revisión se realizó una versión gratuita, y se determinó como una de las desventajas en esta herramienta.

\section{Imagen 1}

Vista de opciones de respuestas - Kahoot

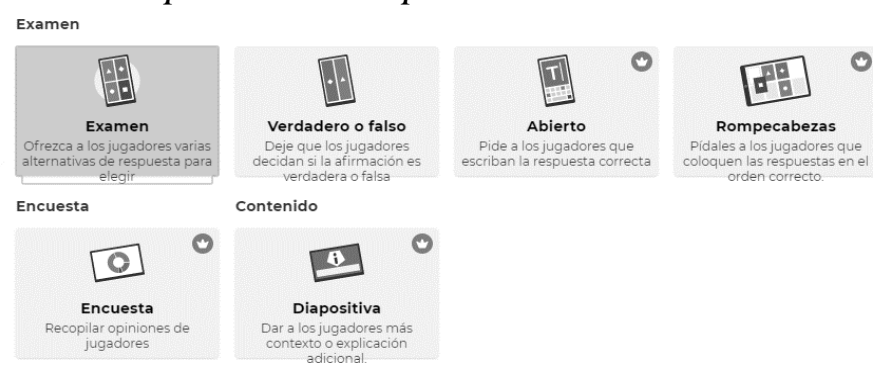

Nota. Opciones de respuestas disponibles en la versión gratuita de Kahoot, tomado de la consulta del 08 de marzo de 2020.

2. Visualización de resultados. Para los docentes, la visualización de resultados es un componente fundamental y esta herramienta cuenta con un apartado donde se pueden ver los reportes al final de clase ya sea en la plataforma o si se prefiere, también permite descargar en un formato Excel. Visualizar los resultados en la plataforma tiene la ventaja de mostrar las preguntas falladas en su mayoría, así como 
también en qué tiempo se resolvieron como se muestra en la Imagen 2. Como desventaja se observó que, en la plataforma no se puede ver detalladamente el reporte debido al control de versiones de paga.

\section{Imagen 2}

Resultados de respuestas correctas.

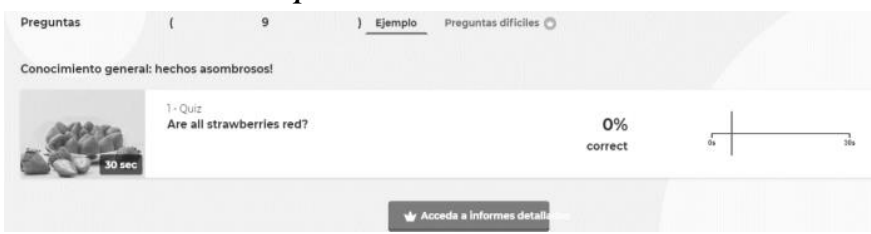

Nota. Vista de la visualización de resultados en la herramienta kahoot, tomado en la consulta del 08 de marzo de 2020.

3. Dispositivos disponibles. Esta herramienta está disponible para todos los dispositivos smartphone, tablet y computadoras tanto Android e ISO. En el caso de las computadoras, a la fecha de la consulta del día 08 de marzo de 2020 se encuentran versiones para los sistemas operativos de Windows 8 y 10 e ISO.

4. Versiones disponibles. Kahoot cuenta con varias formas de poder utilizarla, existe una versión de prueba, pro y premium. Dentro de la versión de prueba te permite elegir dos tipos de preguntas al momento de crear el cuestionario, puedes hacer uso de música, juegos en vivo solo para clases de alumnos (no disponible para asambleas o eventos grandes), elegir del banco de preguntas entre otras cosas. En las versiones de paga permite elegir más opciones de preguntas que se pueden incluir en el cuestionario, hacer eventos con más de 2000 alumnos, agregar videos, imágenes, diapositivas, rompecabezas, entre otros. Kahoot, permite una prueba de 7 días que sirve para evaluar y decidir por alguna de las versiones, sin embargo, entre la versión pro y premium, no hay gran diferencia.

5. Banco de preguntas. Cuenta con una biblioteca de preguntas disponible en caso de que el profesor lo requiera.

6. Responsable de la prueba (test). En el portal del docente, el profesor es el responsable de la clase y es quién puede hacer preguntas.

Socrative. Se propone como una herramienta en entornos digitales de apoyo a los procesos de evaluación en la educación. Cuenta con opciones de preguntas simples, preguntas con tiempo y preguntas con puntuación y los resultados se conocen en tiempo real lo cual permite realimentar inmediatamente a los estudiantes. Algo muy importante con esta herramienta es que te permite diseñar rúbricas lo cual facilita el proceso de evaluación de las actividades y descargarlas a un archivo Excel, PDF y también enviar tus resultados a una cuenta de Google Drive Socrative se puede acceder desde un equipo de cómputo o un dispositivo móvil (tabletas o smartphone) (Socrative, 2011). Su sitio de trabajo se encuentra en https://socrative.com/

A continuación, se muestra el resultado del análisis de la herramienta Socrative de acuerdo con la Tabla 1:

1. Opciones de preguntas. Como resultado de la revisión se tiene que Socrative, permite preguntas con respuestas de opción múltiples, verdadero $\mathrm{o}$ falso y de respuesta corta, igualmente permite combinar entre estas opciones de respuesta en un mismo cuestionario como se muestra en la Imagen 4.

\section{Imagen 3}

Vista de opciones de respuestas - Socrative.

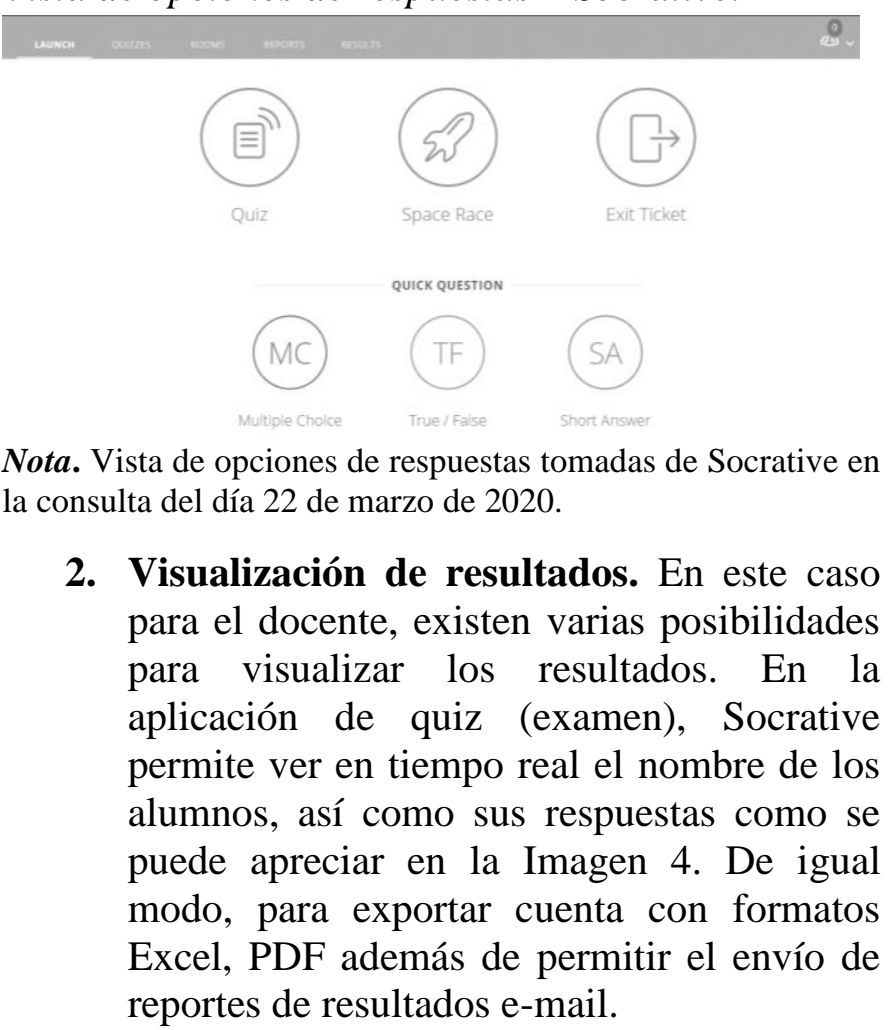




\section{Imagen 4}

Vista de respuestas en tiempo real.
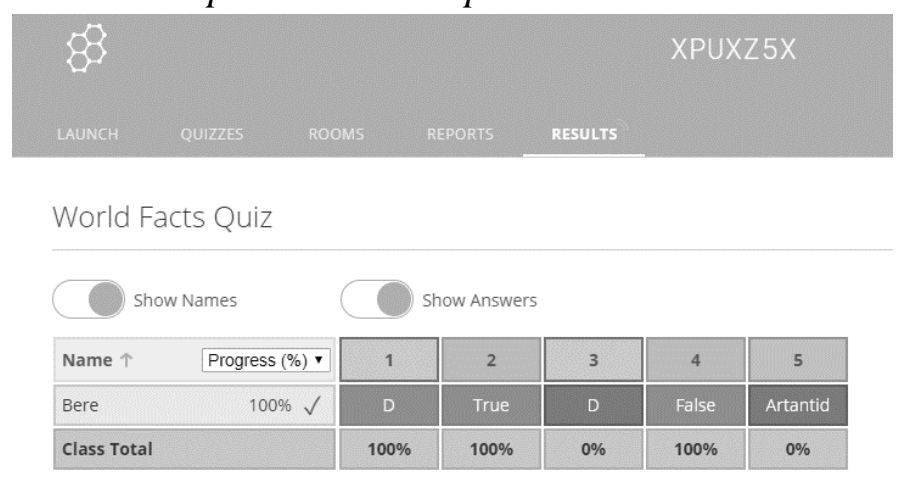

Click question numbers or class total percentages for detailed views.

Nota. Respuestas en tiempo real tomada de la consulta realizada el día 22 de marzo de 2020.

3. Disponibilidad de dispositivos. Esta herramienta está disponible para todos los dispositivos celulares, tabletas o computadoras. Asimismo, ofrece versiones gratuitas para estudiantes o profesores. Las versiones para dispositivos móviles disponibles son Android e ISO, en el caso de los sistemas operativos está disponible para Windows e ISO para computadoras.

4. Versiones disponibles. Los diferentes tipos de planes en esta herramienta como se puede ver en la Imagen 5 son parecidos a la anterior, versiones gratis pro y pro-edu-corporate. El plan gratis de Socrative, en comparación de las otras herramientas, está muy limitada al no contar con otras plantillas creadas sobre alguna temática que pudieran ser modificadas por el profesor. La versión free, para el caso de las actividades, solo está permitido publicar una actividad, eso quiere decir, que el profesor no podrá realizar varios cuestionarios para diferentes equipos. En el plan PRO es donde te permite realizar tus propios cuestionarios con las opciones de preguntas elegidas, de igual modo, este plan sí permite publicar más de una actividad a la vez y puedes importar listas a través de CVS y Excel. Por último, en la versión PROCORPORATE es muy parecido al plan anterior, con la excepción que permite al docente compartir el enlace del cuestionario para facilitar el inicio de sesión del alumno.

\section{Imagen 6}

Versiones de Socrative.

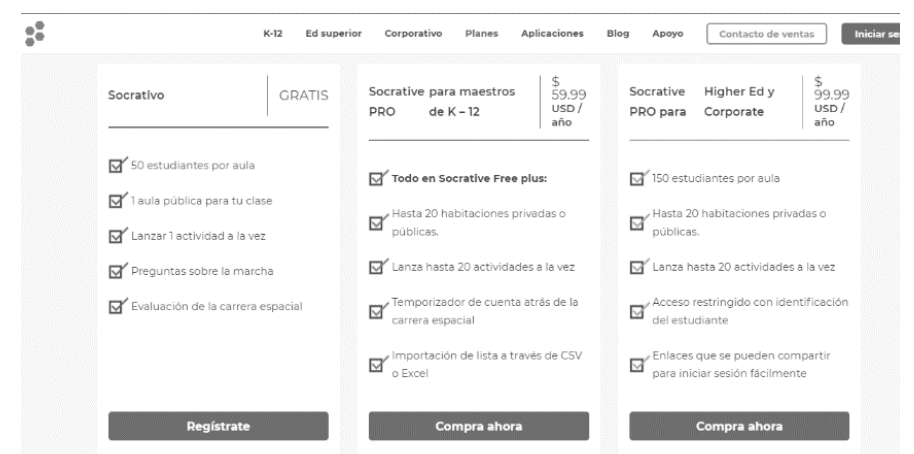

Nota. Versiones de Socrative obtenidas de la consulta del día 22 de marzo de 2020.

5. Banco de preguntas. Socrative ofrece un banco de preguntas o test (prueba) ya disponibles públicamente para su uso.

6. Responsable de la prueba. Para la elaboración de pruebas el profesor es el responsable de la realización y seguimiento de resultados.

Quizizz. Esta herramienta permite el juego de preguntas entre varios jugadores (equipos) con actividades divertidas y entretenidas debido a que cuenta con opciones para personalizar las preguntase o incluso crear preguntas propias para generar exámenes o juegos entre sus alumnos. Para poder jugar, el profesor genera las preguntas y comparte un código que los alumnos ingresarán a su navegador web, con ese código, los alumnos pueden jugar desde su dispositivo móvil o equipo de cómputo. Las preguntas que crea el profesor pueden ser compartidas o privadas e incluir imágenes, audio o vídeo con opción a diferentes modalidades. En el caso de los resultados se pueden enviar a través de otras plataformas e inclusive se puede enlazar a Google Classroom. Es compatible con todos los dispositivos (Quizizz, 2015). Su sitio de trabajo se encuentra en https://quizizz.com/

A continuación, se muestra el resultado del análisis de la herramienta Quizizz de acuerdo con la Tabla 1:

1. Diferentes opciones de preguntas. En esta herramienta el tipo de opciones de preguntas es limitado debido a que solo ofrece preguntas con respuesta única, así como también de selección múltiple como se muestra en la imagen 6. Sin embargo, algo de gran interés es el hecho de permitir agregar a 
Sin embargo, algo de gran interés es el hecho de permitir agregar a las preguntas una fórmula matemática o imágenes esto con el propósito de personalizar las preguntas acordes al tema o área que se está abordando.

\section{Imagen 6}

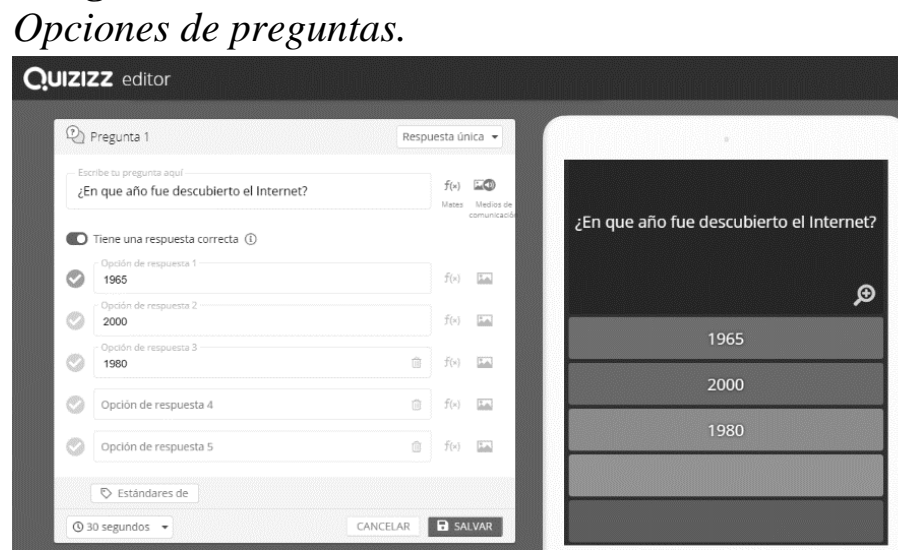

Nota. Vista de opciones de preguntas tomado de la consulta del día 22 de marzo de 2020.

2. Visualización de resultados. Quizziz, permite consultar los resultados en tiempo real o consultarlos en cualquier momento dado que estos resultados están almacenados en la plataforma.

3. Disponibilidad de dispositivos. Esta herramienta está disponible para todos los dispositivos celulares, tabletas y computadoras. Asimismo, ofrece versiones gratuitas para estudiantes y profesores. Las versiones para dispositivos móviles disponibles son Android e ISO, en el caso de los sistemas operativos está disponible para Windows e ISO para computadoras.

4. Versiones disponibles. Esta herramienta no cuenta con planes, es totalmente gratis para los docentes permitiendo acceder a todas las funciones integradas (Imagen 7), con un número de participantes de hasta 12 docentes. Quizzizz, es una buena opción al no tener restricciones para el número de pruebas (quiz).

5. Banco de preguntas. Esta herramienta cuenta con comunidad grandiosa de usuarios quiénes aportan cuestionarios de uso público para todos los docentes, sin embargo, al momento de crear cuestionarios se permite elegir si se desea de uso privado o público

\section{Imagen 7}

\section{Funciones integradas.}

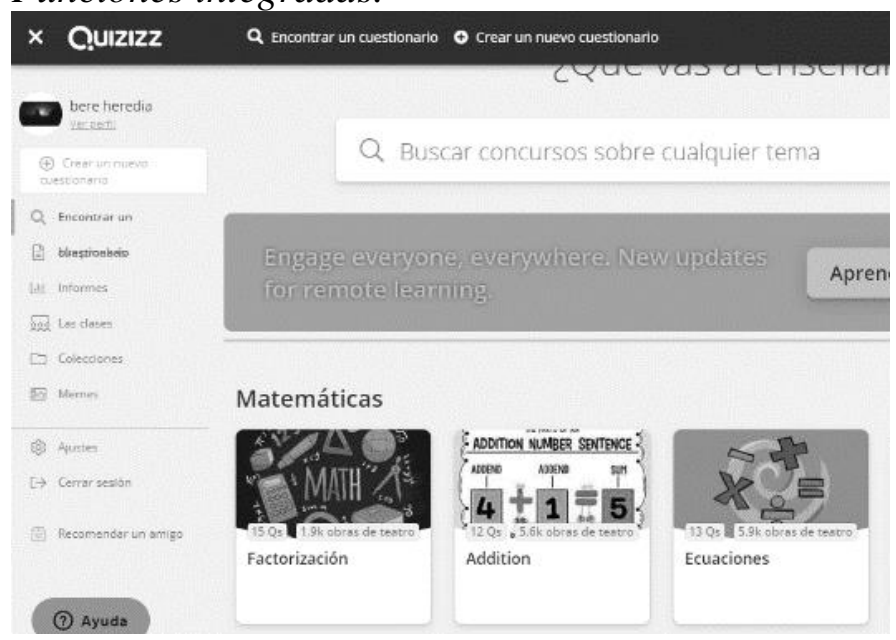

Nota. Vista completa de funciones integradas tomada de la consulta del día 22 de marzo de 2020.

Al dejar los cuestionarios en uso público se crea una biblioteca con cuestionarios listos para usar por cualquier usuario.

6. Responsable de la prueba. Para la elaboración de pruebas, en Quizizz, el profesor es el responsable de la realización y seguimiento de resultados.

\section{Resultado}

Al concluir la revisión de Kahoot, Socrative y Quizizz, se determinó que Quizizz es la herramienta más completa que le permite al docente llevar sus actividades más dinámicas, pues a pesar de ser de acceso libre tiene todos sus elementos disponibles y permite acceder a diversas funcionalidades. Ver tabla 2 que contiene el resultado de estas comparaciones.

El modelo clásico de educación limita la interacción entre docentes, estudiantes y contenidos. En este sentido, la gamificación emerge como como una herramienta pertinente para motivar el desarrollo de contenidos y la participación de los estudiantes en el aula (Villalustre-Martinez \& Del Moral-Pérez, 2015, p.15). Lo anterior confirma que el juego es un proceso positivo que permite combinar modernas metodologías hábiles viables para la educación posibilitando en los alumnos las acciones experimentales, donde pueden recrear, reintentar, observar e innovar para aprender (Álvarez \& Polanco, 2018, p.22). 
Tabla 2

Comparación de tributos Kahoot, Socrative y

Quizizz.

\begin{tabular}{|c|c|c|c|}
\hline Atributo & Kahoot & Socrative & Quizizz \\
\hline $\begin{array}{c}\text { Acceso } \\
\text { gratuito }\end{array}$ & $\checkmark$ & $\checkmark$ & $\checkmark$ \\
\hline $\begin{array}{c}\text { Restricciones } \\
\text { en formato } \\
\text { free }\end{array}$ & $\checkmark$ & $\checkmark$ & $x$ \\
\hline $\begin{array}{c}\text { Acceso } \\
\text { cuenta de } \\
\text { Google }\end{array}$ & $\begin{array}{l}\checkmark \text { cualquier } \\
\text { cuenta de } \\
\text { correo } \\
\text { electrónico }\end{array}$ & $\begin{array}{l}\checkmark \text { cualquier } \\
\text { cuenta de } \\
\text { correo } \\
\text { electrónico }\end{array}$ & $\checkmark$ \\
\hline $\begin{array}{c}\text { Disponible } \\
\text { para } \\
\text { Android y } \\
\text { iOS }\end{array}$ & $\checkmark$ & $\checkmark$ & $x$ \\
\hline $\begin{array}{l}\text { Acceso en el } \\
\text { tiempo }\end{array}$ & Asíncrono & $\begin{array}{l}\text { Síncrono y } \\
\text { Asíncrono }\end{array}$ & $\begin{array}{l}\text { Síncrono y } \\
\text { Asíncrono }\end{array}$ \\
\hline $\begin{array}{c}\text { Cuenta como } \\
\text { alumno }\end{array}$ & $\begin{array}{c}\mathbf{x} \text { acceso con } \\
\text { pin }\end{array}$ & $\checkmark$ & $\begin{array}{c}\times \text { con pin de } \\
\text { acceso }\end{array}$ \\
\hline $\begin{array}{l}\text { Misma clave } \\
\text { o pin de } \\
\text { acceso }\end{array}$ & $\begin{array}{c}\text { x nuevo para } \\
\text { cada } \\
\text { cuestionario }\end{array}$ & $\begin{array}{l}\checkmark \text { mismo parc } \\
\text { cada clase }\end{array}$ & $\begin{array}{c}\checkmark \text { mismo parc } \\
\text { cada clase }\end{array}$ \\
\hline $\begin{array}{l}\text { Opciones de } \\
\text { estilos de } \\
\text { preguntas }\end{array}$ & $\checkmark$ & $\checkmark$ & $\checkmark$ \\
\hline $\begin{array}{l}\text { Respuestas } \\
\text { múltiples }\end{array}$ & $x$ & $\checkmark$ & $x$ \\
\hline $\begin{array}{l}\text { Control del } \\
\text { tiempo en las } \\
\text { preguntas }\end{array}$ & $\checkmark$ limitado & $\checkmark$ configurabl & $\checkmark$ limitado \\
\hline $\begin{array}{l}\text { Preguntas } \\
\text { aleatorias }\end{array}$ & $\checkmark$ & $\checkmark$ & $\checkmark$ \\
\hline Respuesta & Inmediato & $\begin{array}{c}\text { Integra } \\
\text { comentarios e1 } \\
\text { respuestas }\end{array}$ & $\begin{array}{c}\text { Activas o } \\
\text { desactivar la } \\
\text { respuesta } \\
\text { animada }\end{array}$ \\
\hline $\begin{array}{l}\text { Catálogo de } \\
\text { preguntas }\end{array}$ & $\checkmark$ & $\checkmark$ & $\checkmark$ \\
\hline Ritmo & $\begin{array}{l}\checkmark \text { alumno y } \\
\text { profesor }\end{array}$ & $\checkmark$ profesor & $\checkmark$ alumno \\
\hline $\begin{array}{l}\text { Integración } \\
\text { de elementos } \\
\text { multimedios }\end{array}$ & $\begin{array}{l}\checkmark \text { imagen y } \\
\text { vídeo }\end{array}$ & $\checkmark$ imagen & $\checkmark$ imagen \\
\hline $\begin{array}{c}\text { Acceder } \\
\text { computadora } \\
\text { o dispositivo } \\
\text { móvil }\end{array}$ & $\checkmark$ & $\checkmark$ & $\checkmark$ \\
\hline $\begin{array}{l}\text { Dominio de } \\
\text { preguntas }\end{array}$ & $\begin{array}{l}\checkmark \text { básicas y dı } \\
\text { memoria }\end{array}$ & $\begin{array}{c}\checkmark \text { memoria y } \\
\text { pensamiento } \\
\text { avanzado }\end{array}$ & $\begin{array}{c}\checkmark \text { memoria y } \\
\text { pensamiento } \\
\text { avanzado }\end{array}$ \\
\hline Reporte & $\begin{array}{c}\checkmark \text { por } \\
\text { descarga }\end{array}$ & $\begin{array}{l}\checkmark \text { descarga y } \\
\text { vista en línea }\end{array}$ & $\begin{array}{l}\checkmark \text { descarga y } \\
\text { vista en línea }\end{array}$ \\
\hline
\end{tabular}

Nota. Muestra el resultado final de la revisión-comparación de tributos entre Kahoot, Socrative y Quizizz, realizadas para esta investigación.
El modelo clásico de educación limita la interacción entre docentes, estudiantes y contenidos. En este sentido, la gamificación emerge como como una herramienta pertinente para motivar el desarrollo de contenidos y la participación de los estudiantes en el aula (Villalustre-Martinez \& Del Moral-Pérez, 2015, p.15). Lo anterior confirma que el juego es un proceso positivo que permite combinar modernas metodologías hábiles viables para la educación posibilitando en los alumnos las acciones experimentales, donde pueden recrear, reintentar, observar e innovar para aprender (Álvarez \& Polanco, 2018, p.22).

La eficacia de las estrategias de gamificación en educación es avalada por las comparaciones de cada uno de los elementos como lo indican (Dichivela, 2015, p.1), (Hanson-Smith, 2016, p. 230) y (Simões, Díaz-Rendon \& Vilas, 2013, p. 350). Además, permite motivar extrínsecamente el cambio de las conductas negativas de los estudiantes que se ven animados a competir por premios lo cual coincide con la realidad de nuestros jóvenes quiénes invierten muchas horas de su tiempo en juegos síncronos grupales a distancia, por tanto, los docentes pueden aprovechar estas habilidades y canalizarlas en usos formativos y educativos.

De igual modo y en concordancia con (GraciaAretio, 2016, p. 12), los diferentes recursos tradicionales (materiales impresos, audiovisuales e informáticos) han demostrado eficacia potenciando el aprendizaje de los educandos, lo cual se puede ver dado que desde la niñez el juego ha estado implicado en el aprendizaje y en el desarrollo de habilidades de los infantes, por tanto, aplicando nuevas tecnologías en el uso de internet y los videojuegos se puede captar la atención de niños y jóvenes, lograr que su motivación crezca, puesto que no es simplemente jugar, es motivar para aprender. Se puede lograr que aquellas disciplinas consideradas simples o aburridas sean más atractivas, interactivas y de competencia virtual y al mismo tiempo cumplir con los objetivos de la clase.

\section{Conclusiones}

Con los resultados obtenidos en esta investigación y del análisis de comparación entre Kahoot, Socrative y Quizizz, se concluye que son herramientas particularmente útiles que pueden ser aplicadas dentro y fuera del aula apoyando en la 
generación de conocimiento, así como también en el desarrollo de habilidades y destrezas establecidas en los objetivos curriculares. Como resultado de la investigación se tiene que Quizizz, es la herramienta de Gamificación que ofrece mayores beneficios al profesor siendo de software gratuito y fácilmente accesible tanto para los docentes como para los alumnos, por tanto, su implementación en el aula será de acceso rápido, sencillo, sin costos adicionales. Todas estas ventajas permiten que la experiencia docente sea económicamente sostenible y eficiente abriendo un abanico de posibilidades para transferir su uso a cualquier clase o currículo.

De este modo, con la gamificación, es posible centrar el aprendizaje en el alumno brindando facilidades al profesor para efectuar un seguimiento de los estudiantes, registrando sus avances, aumentando el grado de motivación e interactividad y al mismo tiempo fomentando la participación de los estudiantes en el aula. Estas nuevas aplicaciones de gamificación vienen en un entorno de trabajo simple, intuitivo y amigable, principalmente para los más jóvenes.

Por tanto, en esta nueva forma de enseñar, corresponde al profesor activar el aprendizaje de sus estudiantes con el conocimiento de las habilidades y virtudes, así como también aplicando su creatividad en el diseño de actividades pensadas en generar un aprendizaje significativo de tal forma que a manera de juego se logre captar la atención de los estudiantes y los motive a enrolarse en sus propios aprendizajes.

Por último, en estas herramientas de gamificación las actividades competitivas están consideradas como una de las principales mecánicas de gamificación en el aula siempre que estén bien organizadas y no se centren en los resultados concretos de la competencia y los rete a dar lo mejor de ellos mismos.

Las nuevas formas de enseñanza que integran los recursos tecnológicos y de gamificación, engloban un compromiso social entre universidades y gobierno en donde los actores principales están por un lado el profesor como guía en el proceso de aprendizaje y por el otro, al alumno como protagonista de su propio aprendizaje.

\section{Referencias}

Álvarez, A., \& Polanco, N. (2018). La gamificación como experiencia de aprendizaje en la educación. Revista Tecnólogica-Educativa Docentes 2.0, 6(3), $39 . \quad$ Recuperado de https://ojs.docentes20.com/index.php/revista-
Ara-Moreira, M., \& González-González , C. (2015). De la enseñanza con libros de texto al aprendizaje en espacios online gamificados. Universidad de Murcia, XXXIII(3), 15-38. Recuperado de: http://hdl.handle.net/10201/49470

Carrión-Candel, E. (2018). El uso de la Gamificación y los recursos digitales en el aprendizaje de las Ciencias Sociales en la Educación Superior. DIM: Didáctica, Innovación y Multimedia (36), 14. Recuperado de: http://dimglobal.net/revista.htm

Deterding, S. (011). Gamification: Toward a Definition. Hans Bredow Institute for Media, 4. doi:http://gamification-research.org/wpcontent/uploads/2011/04/02-Deterding-Khaled-NackeDixon.pdf

Dichivela, D. (2015). Gamification in Education: A Systematic Mapping Study. Educational Technology \& Society, 75-88.

Gálvez-Sosa, M. I., \& Rodríguez-Ledezma, N. C. (2005). La importacia del juego (Cap III). Tesis, Universidad de las Américas Puebla, Departamento de Diseño de Gráfico. Escuela de Artes y Humanidades, Cholula, Puebla. Recuperado de: http://catarina.udlap.mx/u_dl_a/tales/documentos/ldf/galvez_s _mi/

García-Aretio, L. (2016). El juego y otros principios pedagógicos. Su pervivencia en la educación a distancia y virtual. (A. I. Distancia, Ed.) La Revista Iberoamericana de la Educación Digita - RIED, XIX(2), 9-23. doi:DOI: http://dx.doi.org/10.5944/ried.19.2.16175

Green, C., \& Bavelier, D. (2006). Effect of action video games on the spatial distribution of visuospatial attention. Journal of Experimental Psychology: Perception and Performance, 6(32), 1465-1478. doi:10.1037/0096-1523.32.6.1465

Hanson-Smith, E. (2016). Games, Gaming, and Gamification: Some Aspects of Motivation. TESOL journal, 227-232. doi:https://onlinelibrary.wiley.com/doi/abs/10.1002/tesj.233

Hernández-Sampieri, R., Fernández-Collado, C., \& Baptista-Lucio, M. (2014). Metodología de la investigación. (6ta edición ed.). McGraw-hill

Kahoot. (2013). Kahoot Juego de Video. Recuperado de: https://kahoot.com/schools-u/

López-Jiménez, D. F. (2007). La naturaleza de las tecnologías de información y comunicación: las TIC como determinantes de la organización y de la sociedad de la información. Palabra Clave, 10(1), 72-93. Recuperado de: http://dspace.uhemisferios.edu.ec:8080/xmlui/handle/1234567 $89 / 453$

Mujica, R. (2020). Fundamentos de la Tecnología Educativa. Revista Tecnológica-Educativa Docentes 2.0, 8(1), 15-20. doi:https://doi.org/10.37843/rted.v8i1.82

Müller-Ponce, M. (2019). La educación de las virtudes humanas. El profesor como modelo de virtud para sus. Facultad de Artes y Humanidades, Psicopedagogía. Quito: Universidad de los Hemisferios. Recuperado de http://dspace.uhemisferios.edu.ec:8080/xmlui/handle/12 $3456789 / 860$ 
Oliva, H. A. (2017). La gamificación como estrategia metodológica en el contexto educativo universitario. Realidad $y \quad$ Reflexion(44), 29-47. doi:https://doi.org/10.5377/ryr.v44i0.3563

Palazón, J. (2019). ¿Qué es la gamificación y cuáles son sus objetivos? Recuperado de: https://www.educaciontrespuntocero.com/noticias/gamif icacion-que-es-objetivos/

Palazón, J. (2020). 25 herramientas de gamificación para clase que engancharán a tus alumno. Recuperado de: https://www.educaciontrespuntocero.com/recursos/herra mientas-gamificacion-educacion/

Quizizz. (2015). Quizziz Desarrollador de software. Recuperado de: https://quizizz.com/

Rodrigez, M. D. (2010). Una renovación en el proceso de enseñanza $y$ aprendizaje. Recuperado de: https://www.gestiopolis.com/una-renovacion-procesoensenanza-aprendizaje/

Rodríguez, F., \& Santiago-Campión, R. (2015). Gamificación: Cómo motivar a tu alumnado y mejorar el clima en el aula. Grupo Océano.

Simões, J., Díaz-Rendon, R. \& Vilas, A. F. (2013). A social gamification framework for a K-6 learning platform. Computers in Humans Behavior, XXIX, 345-353. doi:https://doi.org/10.1016/j.chb.2012.06.007

Socrative. (2011). Socrative Aplicación. Recuperado de: https://socrative.com/

Vergara-Rodríguez, D., \& Gómez-Vallecillo, A. I. (2017). Origen de la gamificación educativa. Recuperado de: http://espacioeniac.com/origen-de-la-gamificacioneducativa-por-diego-vergara-rodriguez-y-ana-isabelgomez-vallecillo-universidad-catolica-de-avila/

Villalustre-Martínez, L., \& Del Moral-Pérez, M. E. (2015). Gamificación: Estrategia para optimizar el proceso de aprendizaje y la adquisición de competencias en contexto universitarios. Digital Education Review(27), 13-23. Recuperado de: https://revistes.ub.edu/index.php/der/article/viewFile/11 $591 / \mathrm{pdf}$ 